\title{
Nocardiosis in ectopic ACTH syndrome: A case report and review of 11 cases from the literature
}

\author{
LICHEN XU ${ }^{1,2^{*}}$, QIAOMAI XU ${ }^{1,2^{*}}$, MEIFANG YANG ${ }^{1,2}$, HAINV GAO $^{1,2}$, MINGZHI XU $^{3}$ and WEIHANG MA ${ }^{1,2}$ \\ ${ }^{1}$ State Key Laboratory for Diagnosis and Treatment of Infectious Diseases, The First Affiliated Hospital, \\ Zhejiang University School of Medicine; ${ }^{2}$ The Collaborative Innovation Center for Diagnosis and \\ Treatment of Infectious Diseases; ${ }^{3}$ Department of Endocrinology, The First Affiliated Hospital, \\ Zhejiang University School of Medicine, Hangzhou, Zhejiang 310003, P.R. China
}

Received August 4, 2015; Accepted August 30, 2016

DOI: $10.3892 / \mathrm{etm} .2016 .3846$

\begin{abstract}
Ectopic adrenocorticotropic hormone (ACTH) syndrome (EAS) associated with nocardiosis is rare, and little information is available regarding its clinical characteristics. In this study, the case of a 35-year-old male patient who showed significant cushingoid features and had a cough with yellow phlegm for 1 month is described. Pulmonary computed tomography (CT) scanning and ${ }^{18} \mathrm{~F}$-fluorodeoxyglucose positron emission tomography combined with $\mathrm{CT}$ identified two different lesions in the mediastinum and pulmonary region, respectively. The lesion in the mediastinum was finally diagnosed as an ACTH-secreting mediastinal paraganglioma via biopsy. The sputum culture confirmed pulmonary nocardiosis. The patient was effectively treated with complete tumor resection following the treatment of nocardiosis using trimethoprim-sulfamethoxazole. Following the present case, 11 additional cases of nocardiosis in EAS were identified in the literature and their clinical characteristics were compared and evaluated. It may be concluded that, although Nocardia remains a rare opportunistic infection pathogen in EAS, it is necessary to consider nocardiosis as a diagnosis for patients with pulmonary imaging findings of cavity, consolidation or nodule, particularly when there are brain and extra-pulmonary lesions as well as a poor response to regular treatment.
\end{abstract}

Correspondence to: Dr Weihang Ma, State Key Laboratory for Diagnosis and Treatment of Infectious Diseases, The First Affiliated Hospital, Zhejiang University School of Medicine, 79 Qingchun Road, Hangzhou, Zhejiang 310003, P.R. China

E-mail: weihangma2014@126.com

*Contributed equally

Key words: nocardiosis, ectopic adrenocorticotropic hormone syndrome, paraganglioma, mediastinum

\section{Introduction}

Endogenous Cushing syndrome (ECS) results from lengthy and inappropriate exposure to excessive levels of glucocorticoid secretion, and is generally divided into adrenocorticotropic hormone (ACTH)-dependent and ACTH-independent. Ectopic ACTH syndrome (EAS) is characterized by hypercortisolemia as a result of extra-pituitary ACTH secretion and accounts for $20 \%$ of ACTH-dependent Cushing syndrome cases (1).

Nocardia spp., a gram-positive bacterium, causes local or disseminated infection in humans and animals. Patients with depressed cell-mediated immunity are at high risk for infection, including those with solid-organ or hematopoietic stem cell transplantation, human immunodeficiency virus infection, long-term steroid use or malignancy (2). Although Nocardia has been considered to be rare, a previous report has shown that its incidence is increasing (3). Extremely high glucocorticoid doses in patients with ECS affect virtually every cell type involved in immunity and the inflammatory response, particularly cell-mediated immunity, which causes such patients to be a target of nocardiosis (4). However, the clinical features of few cases of nocardiosis in EAS have been documented.

In the present study, a case of rare ectopic ACTH-secreting paraganglioma in the mediastinum associated with nocardiosis is presented. In addition, the clinical features of 11 published cases of EAS associated with Nocardia infection were analyzed (5-11). The aim of this study was to provide guidance for the clinical diagnosis and treatment of Nocardia infection associated with EAS.

\section{Case report}

The patient and their family were informed that data from the case would be submitted for publication and provided consent accordingly. A 35-year-old male patient first presented to the First Affiliated Hospital, Zhejiang University School of Medicine (Hangzhou, China) in February 2012, with weakness, polyuria and polydipsia for 7 years and had been diagnosed as having hypertension with unsatisfactory drug control for 6 months. Frequently, the patient suffered from blurred vision, headache and limb numbness. One month prior to presentation, he had developed a cough with dark yellow phlegm 
without fever and dyspnea. The patient was transferred to this hospital to obtain a definite diagnosis. Physical examination on admission found the patient to have a blood pressure of $172 / 102 \mathrm{mmHg}$, heart rate of 80 beats/min with occasional arrhythmia, body mass index of $25 \mathrm{~kg} / \mathrm{m}^{2}$, and normal body temperature. The patient exhibited a moon face, buffalo hump, polycythemia, edema of the feet and central obesity. Other positive findings included purple striae in the abdomen, acne, and a dark skin color. There were no clinically palpable nodules. Other systemic examination findings were normal.

The initial laboratory evaluation revealed hypokalemia (K level, $2.48 \mathrm{mmol} / 1$; reference, 3.50-5.20 $\mathrm{mmol} / \mathrm{l}$ ), hyperglycemia (fasting plasma glucose, $10.97 \mathrm{mmol} / \mathrm{l}$; reference, 3.9-7.8 mmol/1) and hyperlipidemia (triglycerides, $4.41 \mathrm{mmol} / \mathrm{l}$; reference, 0.3-1.7 $\mathrm{mmol} / \mathrm{l})$. Blood routine tests showed a normal leukocyte count leukocyte count $\left(8.1 \times 10^{9} / \mathrm{L}\right.$; reference, $\left.4-10 \times 10^{9} / \mathrm{L}\right)$ but mildly increased neutrophil percentage (83.6\%; reference, 50-70\%). A hormonal assessment panel was performed, which revealed markedly increased urinary $(3,118.08 \mu \mathrm{g} / 24 \mathrm{~h}$; reference, 55.5-286.0 $\mu \mathrm{g} / 24 \mathrm{~h})$ and serum (>50 $\mu \mathrm{g} / \mathrm{dl}$; reference, 5.0-25.0 $\mu \mathrm{g} / \mathrm{dl})$ cortisol and serum ACTH (372 pg/ml; reference, 0.0-46.0 pg/ml) levels.

High-dose dexamethasone suppression test: The measurement of serum cortisol between 8:00 and 9:00 AM before and after a high-dose $(8 \mathrm{mg})$ oral night intake of dexamethasone and is considered suggestive of suppression when a reduction of $>50 \%$ is observed compared to the baseline value.

Low-dose dexamethasone suppression test was performed after an overnight oral intake (between 11:00 PM and 12:00 AM) dexamethasone (1 mg), and blood collection for measurement of serum cortisol should occur in the subsequent morning between 8:00 and 9:00 AM. Cortisol values above $1.8 \mu \mathrm{g} / \mathrm{dL}$ are considered abnormal. Cortisol levels were not suppressed by high- or low-dose dexamethasone.

To investigate the symptoms of cough and expectoration, chest computed tomography (CT; Fig. 1) scanning was performed. The resulting image showed a soft tissue shadow in the anterior mediastinum and multiple nodules with a partial cavity lesion in bilateral lung fields. Brain magnetic resonance imaging (MRI) revealed a slightly swollen pituitary gland, but this MRI result was not able to explain the patient's clinical manifestations. Laboratory tests, and the MRI and CT scans supported a preliminary diagnosis of ectopic ACTH-secreting tumor. However, it was not possible to determine whether the multiple lesions in the lung constituted an infection focus or metastasis focus. For further clarification, the patient underwent ${ }^{18} \mathrm{~F}$-fluorodeoxyglucose $\left({ }^{18} \mathrm{~F}-\mathrm{FDG}\right)$ positron emission tomography (PET) combined with CT (Fig. 2). The resulting imaging showed a tumor focus with mildly increased ${ }^{18} \mathrm{~F}$-FDG metabolism in the anterior mediastinum. Multiple clear-boundary nodular changes in the bilateral pulmonary region suggested possible infection. Histopathological analysis revealed tumor cells arranged in nests. Immunohistochemistry showed that the periphery of the cell nests was positive for chromogranin A and S-100. Immunohistochemistry analysis was performed using a Dako Autostainer with Envision ${ }^{\mathrm{TM}}$ Detection Kit, chromogranin A (DAK-A3; 1:200) and polyclonal S-100 (1:300; Dako Denmark A/S, Glostrup, Denmark). Those microscopy findings confirmed paraganglioma.
Treatment was initiated while the imaging and laboratory examinations were ongoing. According to his manifestations, the patient was initially treated with potassium replacement (oral potassium chloride solution, $60 \mathrm{ml} /$ day), insulin (mixed protamine zinc recombinant human insulin injection adjusted according to plasma glucose), intravenous fluids (Ringer's solution; adjusted according to intake and output record) and anti-hypertension medication (amlodipine, $5 \mathrm{mg} / \mathrm{day}$; perindopril, $4 \mathrm{mg} /$ day). As the patient had symptoms of cough and expectoration, antibiotics (cefoperazone/sulbactam; dosage, $2 \mathrm{~g}$; q8h) were administered after admission, but there was no improvement in the symptoms after 5 days. Following discussion with a doctor of infectious diseases and consideration of the pulmonary CT scan results, a possible diagnosis of pulmonary aspergillosis could not be eliminated. Caspofungin (March 6, 2012; 20 days after admission) at a dosage of $50 \mathrm{mg} /$ day, combined with a formal antibiotics regimen was recommended. Gram staining and sputum culture were performed concurrently. Gram staining of the sputum on March 9, 2012 (23 days after admission) revealed thin-beaded, gram-positive branching rods and modified acid-fast staining was then performed, according to previously described methods (12). The finding that these branching rods were partially acid-fast positive was suggestive of infection with Nocardia. The subsequent culture confirmed this result. According to the clinical, radiological and etiological findings, pulmonary nocardiosis was identified. In consideration of the possibility of co-infection with Aspergillus, a new antibiotic treatment was initiated with oral trimethoprim-sulfamethoxazole (TMP-SMZ) at a dosage of $15 \mathrm{mg} / \mathrm{kg} /$ day (960 mg/day) and intravenous infusion of caspofungin at a dosage of $50 \mathrm{mg} /$ day. Approximately 1 week after starting the new regimen, the patient's condition had improved. At 17 days after antibiotic adjustment, a pulmonary CT scan showed a clear reduction of the bilateral pulmonary foci, which strengthened the diagnosis of infectious lesions in the lungs. Caspofungin was discontinued because the rapid lung lesion shrinkage in the CT images did not support aspergillosis. Furthermore, the Aspergillus antigen levels were within the normal ranges and no evidence in the microscopic examination indicated Aspergillus. Following stabilization of hypokalemia, hyperglycemia, hypertension and infection at 43 days after admission, the patient was transferred to the Department of Thoracic Surgery. The mass in the anterior mediastinum and a nodule in the left upper pulmonary lobe were resected. Pathological evaluation suggested a chronic inflammatory focus in left upper pulmonary lobe with no evidence of malignancy. The pathological features and immunohistochemical observations for the mass in the anterior mediastinum indicated paraganglioma and corresponded with the result of formal biopsy.

At 3 days after the surgery, the plasma ACTH level dropped shapely to $12.7 \mathrm{pg} / \mathrm{ml}$, which was within the normal range. The patient was discharged without any postoperative complications and continued oral treatment with TMP-SMX for 6 months. A CT scan 4 months after surgery indicated the lung lesion had been replaced by fibrous striped shadows without signs of recurrence. There was no recurrence of either nocardiosis or paraganglioma during a 3-year follow-up. 
A

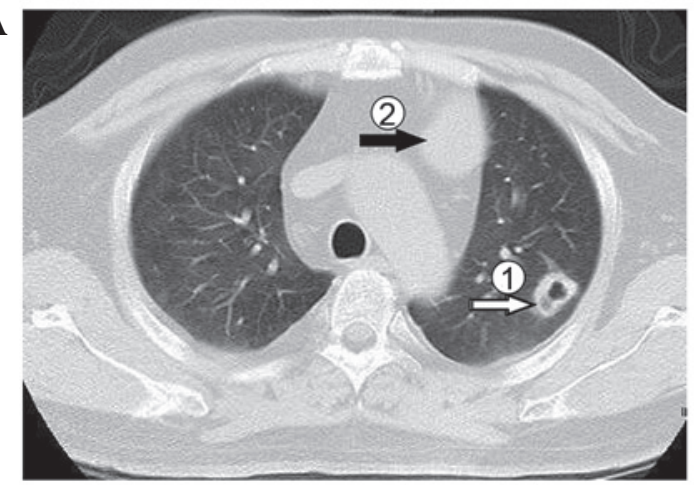

B

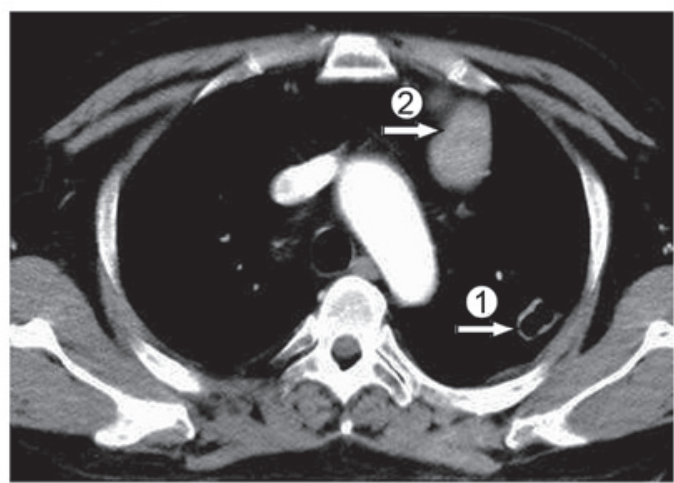

Figure 1. Multiple nodules in bilateral lung fields and a cavity lesion are visible with (A) partial nodular enhancement (arrow 1) and (B) obvious enhancement (arrow 1). A soft tissue density mass can be identified in the anterior mediastinum, adjacent to the aortic arch, with (A) a clear boundary (arrow 2) and heterogeneous enhancement (arrow 2).

A

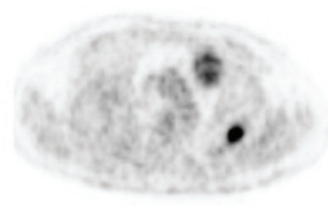

D

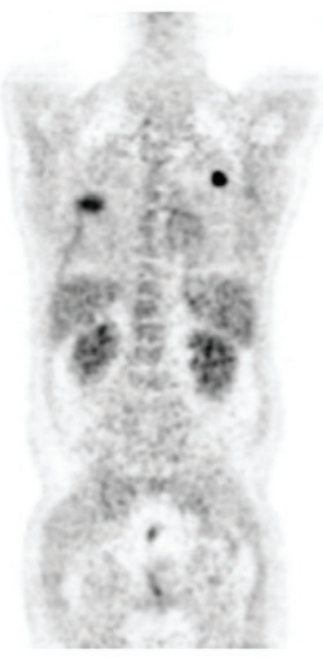

B

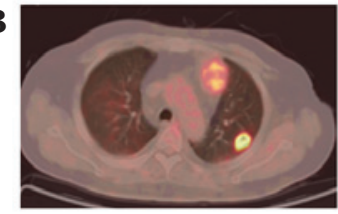

$\mathbf{E}$

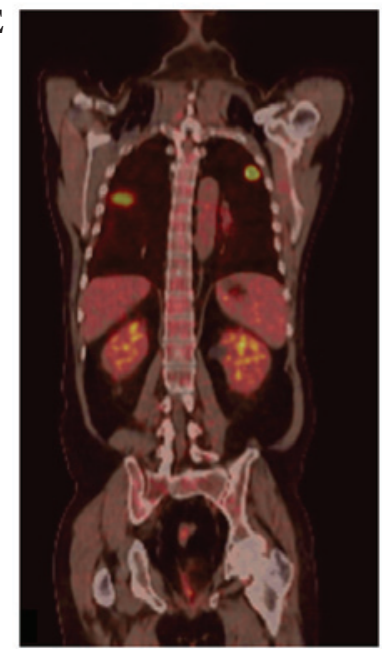

C

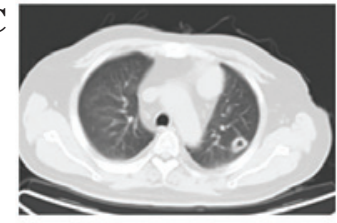

F

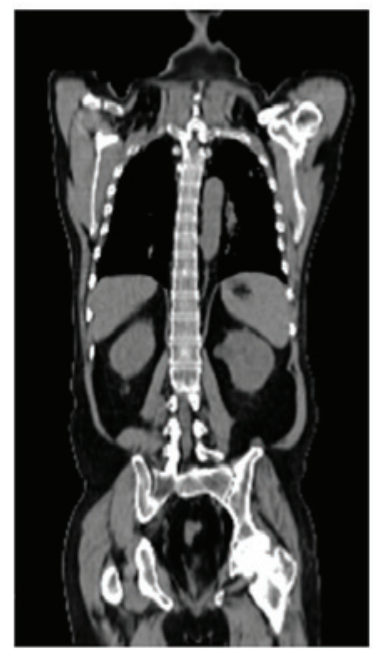

Figure 2. (A-C) Coronal and (D-F) transverse planes of (A and D) ${ }^{18}$ F-FDG PET and (B and E) ${ }^{18}$ F-FDG PET/CT showed focuses increase ${ }^{18}$ F-FDG metabolism in the anterior mediastinum (SUV=4.42) and in bilateral pulmonary (left upper pulmonary nodule, SUV=10). (C and F) Computed tomography scan showed a homogeneous tumor-like focus in the anterior mediastinum and multiple clear-boundary nodule changes in bilateral pulmonary images. ${ }^{18} \mathrm{~F}-\mathrm{FDG}$ PET/CT, ${ }^{18}$ F-Fluorodeoxyglucose positron emission tomography/computed tomography; SUV, standardized uptake value.

\section{Discussion}

The English-language literature published from January 1980 to January 2014 (34 years) in the PubMed database, Google Scholar and Web of Science database was searched using the key words 'Nocardia', 'Nocardia infection', 'Nocardiosis' and 'ACTH Syndrome, Ectopic', 'Ectopic ACTH Syndrome', 'Ectopic ACTH Syndromes'. In addition, the references in the articles that referred to nocardiosis and ectopic ACTH syndrome were also examined. A total of 9 articles with full-text describing 11 cases were available (5-14). Including the present case, the clinical characteristics of EAS with Nocardia infection in 12 cases were analyzed. Information concerning the demography and clinical characteristics of the cases are summarized in Xs I and II.
The literature review found that EAS complicated with nocardiosis was more common in men (9 cases, 75\%) than in women. This result is consistent with previous studies of Nocardia spp. infection, which have reported that males are more susceptible to infection than females $(15,16)$. However, a survey of cases of EAS indicated that men constitute $40-50 \%$ of patients (17). The mean age at diagnosis was 48 years, which falls within the range of mean ages reported previously $(17,18)$.

Typical cushingoid features were readily observed in these cases. The majority of patients exhibited skin changes and muscle weakness, which are often observed in cases of exposure to long-term and extra high-dose glucocorticoids. Those manifestations may indicate that patients with EAS associated with nocardiosis tend to have chronic disease. The 24-h free urine cortisol concentration in the 12 cases examined in the 
Table I. Clinical symptoms and signs of patients with ectopic ACTH syndrome $(n=12)$.

\begin{tabular}{|c|c|}
\hline Variable & Value \\
\hline Mean age (range), years & $48(24-72)$ \\
\hline \multicolumn{2}{|l|}{ Gender, n (\%) } \\
\hline Female & $3(25.00)$ \\
\hline Male & $9(75.00)$ \\
\hline \multicolumn{2}{|l|}{ Hypertension, n (\%) } \\
\hline Yes & $6(50.00)$ \\
\hline No & $5(41.67)$ \\
\hline Not reported & $1(8.33)$ \\
\hline \multicolumn{2}{|l|}{ Weakness, n (\%) } \\
\hline Yes & $9(75.00)$ \\
\hline No & $2(16.67)$ \\
\hline Not reported & $1(8.33)$ \\
\hline \multicolumn{2}{|l|}{ Hirsutism, n (\%) } \\
\hline Yes & $3(25.00)$ \\
\hline No & $8(66.67)$ \\
\hline Not reported & $1(8.33)$ \\
\hline \multicolumn{2}{|l|}{ Skin change ${ }^{\mathrm{a}}, \mathrm{n}(\%)$} \\
\hline Yes & $9(75.00)$ \\
\hline No & $2(16.67)$ \\
\hline Not reported & $1(8.33)$ \\
\hline \multicolumn{2}{|l|}{ Central obesity, n (\%) } \\
\hline Yes & $5(41.67)$ \\
\hline No & $6(50.00)$ \\
\hline Not reported & $1(8.33)$ \\
\hline \multicolumn{2}{|l|}{ Edema, n (\%) } \\
\hline Yes & $2(16.67)$ \\
\hline No & $9(75.00)$ \\
\hline Not reported & $1(8.33)$ \\
\hline \multicolumn{2}{|l|}{ Body weight, n (\%) } \\
\hline Increase & $3(25.00)$ \\
\hline Decrease & $5(41.67)$ \\
\hline Not reported & $4(33.33)$ \\
\hline \multicolumn{2}{|l|}{ Psychiatric disorders, n (\%) } \\
\hline Yes & $1(8.33)$ \\
\hline No & $10(83.33)$ \\
\hline Not reported & $1(8.33)$ \\
\hline \multicolumn{2}{|l|}{ Infections, n (\%) } \\
\hline Nocardia & $12(100.00)$ \\
\hline Lung involved & $12(100.00)$ \\
\hline Brain involved & $1(8.33)$ \\
\hline Skin involved & $2(16.67)$ \\
\hline Opportunistic pathogen co-infection ${ }^{\mathrm{b}}$ & $4(33.33)$ \\
\hline Pneumocystis carinii & $3(25.00)$ \\
\hline Aspergillosis & $2(16.67)$ \\
\hline
\end{tabular}

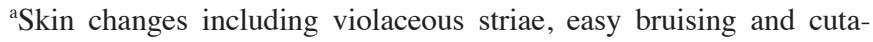

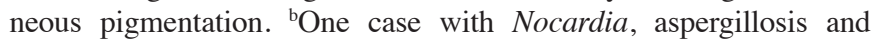
Pneumocystis carinii triple infection. ACTH, adrenocorticotropic hormone. present case was apparently higher than in certain previous reports $(19,20)$. However, restricted by the limited number of cases, it is not possible to determine the exact association of elevated 24-h free urine cortisol concentration with nocardiosis. The level of cortisol determines the degree of immunosuppression. The greater the extent by which cortisol levels exceed the normal value, the greater the possibility of opportunistic infections and the higher the mortality rate (21). The present review demonstrated that, of the 12 cases involving Nocardia spp., the patients who had a fatal outcome as a result of serious infection had comparatively higher urinary levels of corticoids. In addition, patients with higher urine cortisol concentrations tended to be co-infected with other opportunistic pathogens, especially Aspergillus and Pneumocystis carinii. Thus, the possibility of co-infection should be considered for patients with high level of corticoids and a combination antibiotic regimen applied as early as possible.

Radiography is a helpful diagnostic tool in localizing primary tumors and identifying infection lesions. For EAS patients, chest imaging is necessary to identify infection, particularly for individuals without pulmonary symptoms. Imaging results of the 12 cases showed the lung was the most common site of nocardiosis and was involved in all cases included in this literature review. Chest imaging is crucial in providing evidence of Nocardia spp. infection. Although a variety of radiological findings, such as cavities, consolidations/infiltration, nodules/masses and pleural effusion were present in the current case and previous patients in chest CT or X-ray images, the main findings comprised cavity (6 cases, 50\%) and consolidation/infiltration (5 cases, $42 \%)$. Of note, the 4 cases $(33 \%)$ of mass/nodule tended to be multiple. In a previous study of nocardiosis involving 51 cases performed by Blackmon et al, it was shown that airspace consolidation and discrete nodules were the most common manifestations while cavities were found in 21 cases (39.6\%) (22). The high rate of cavities in the present review may result from delayed diagnosis, due to the low incidence and variable manifestations of nocardiosis in EAS. Previous data indicated that cavity formation mostly occurred within 2 weeks of infection (23). The mean time to establish a diagnosis of nocardiosis may be as long as 42 days (24). Formation of a cavity may be facilitated by a prolonged disease course. In conclusion, cavity lesions and consolidation/infiltration were the major findings of pulmonary nocardiosis for patients with EAS in the present study. Accordingly, nocardiosis should be considered as a differential diagnosis for EAS patients who have cavity formation or consolidation nodules in the lung, particularly when these manifestations are associated with brain and extra-pulmonary lesions. It is noteworthy that the imaging features of pulmonary nocardiosis in EAS patients are nonspecific and often mimic malignancy or other infection such as fungal infection and tuberculosis (19). In the present case and another previous study (25), PET/CT imaging showed that nocardiosis is often indicated by a high uptake of ${ }^{18} \mathrm{~F}-\mathrm{FDG}$, which is similar to that in malignant lesions. In this situation, a combination of PET/CT and image-guided biopsy can be a useful supplementary method for making a differential diagnosis.

In general, patients from the literature with only pulmonary infection exhibited sensitivity to TMP-SMZ monotherapy. 


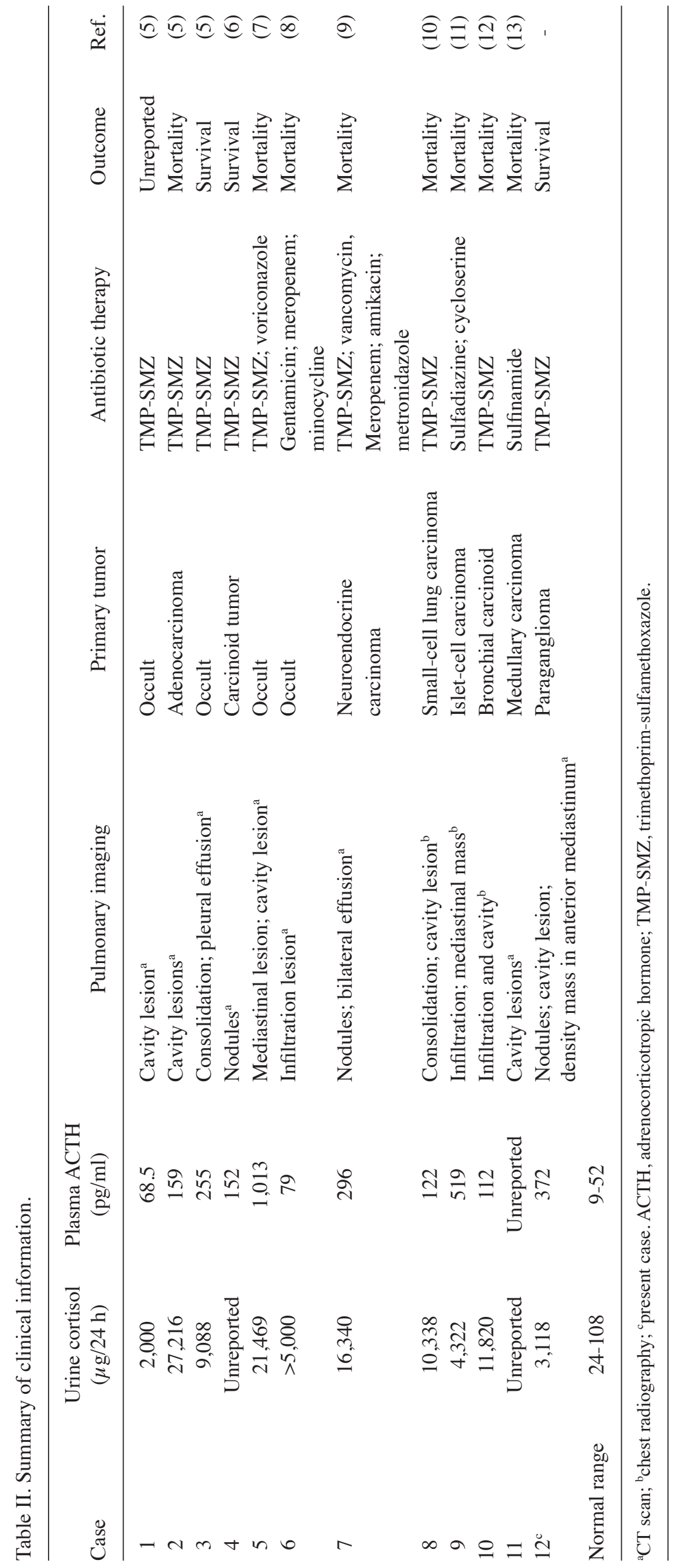


Considering the high incidence and poor prognosis of Aspergillus co-infection, an antibiotic regimen containing an antifungal drug is recommended for early application since it is not possible to eliminate a diagnosis of fungal infection. However, TMP-SMX monotherapy appears to be inadequate in severe or disseminated cases $(8-10,14)$. Although optimal antimicrobial treatment regimens were not firmly established, combined antibiotic therapy with a sulfa-containing agent has been recommended for severe or systematic disease (26). A combination of amikacin and imipenem or broad-spectrum cephalosporin was more effective than TMP-SMZ monotherapy in an experimental mouse model (27). However, a combination of imipenem and amikacin was poorly tested in patients, which is inadequate for drawing a conclusion. A three-drug regimen comprised of TMP-SMZ, amikacin, and either ceftriaxone or imipenem has been recommended when there is no evidence of resistance (28). Also, linezolid as an effective alternative has been reported in several cases of nocardiosis (29). Moreover, differing antimicrobial susceptibility patterns for different Nocardia species will induce varying therapeutic effects. Therefore, susceptibility tests provide significant information for adjustment of the antibiotic, particularly in cases insensitive to initial empirical treatment. Generally, a treatment duration of 6 months is recommended for EAS patients with pulmonary or cutaneous infection and 12 months for those with central nervous system infection or dissemination.

Controlling the plasma level of cortisol not only can improve clinical manifestation but also help in the prevention of Nocardia spp. infection. A study of opportunistic infection indicated that 11 of $12(92 \%)$ patients succumbed when hypercortisolemia was not controlled, whereas 11 of $19(58 \%)$ survived with effective control of hypercortisolemia (30). Complete tumor resection is a direct and effective curative method whenever possible, yet the success rate is only $30-47 \%$ (19). Medical therapy to control cortisol overproduction has been offered to those who are not indicated for complete resection. Metyrapone, ketoconazole and mitotane can all be used to lower plasma cortisol by acting directly to inhibit synthesis and secretion in the adrenal gland (31). These drugs are not effective as solo long-term treatments and are mainly used as preoperative preparations or as an adjunctive therapy after surgery (31). When the cortisol level cannot be controlled properly, when drug treatment is ineffective or not well tolerated or the resource remains unclear after long-term follow-up, total bilateral adrenalectomy will reduce cortisol levels and rapidly resolve clinical features. Following surgery, long-term treatment with glucocorticoids is necessary.

In conclusion, male patients are more vulnerable to infection. Patients with EAS who have extra-high urine cortisol levels and with a long disease course are at higher risk for Nocardia spp. infection. CT presentations of pulmonary nocardiosis in EAS at the time of diagnosis were heterogeneous. Cavity (50\%), consolidation/infiltration (42\%) and nodule/mass (33\%) lesion were three common imaging findings in the present review. Optimal antimicrobial treatment regimens have not been firmly established. TMP-SMZ remains the first choice in EAS associated with pulmonary nocardiosis empirically, while combined antibiotic therapy with a sulfa-containing agent is recommended for severe or disseminated cases.

\section{References}

1. Alexandraki KI and Grossman AB: The ectopic ACTH syndrome. Rev Endocr Metab Disord 11: 117-126, 2010.

2. Minero MV, Marín M, Cercenado E, Rabadán PM, Bouza E and Muñoz P: Nocardiosis at the turn of the Century. Medicine (Baltimore) 88: 250-261, 2009.

3. Beaman BL and Beaman L: Nocardia species: Host-parasite relationships. Clin Microbiol Rev 7: 213-264, 1994.

4. Lionakis MS and Kontoyiannis DP: Glucocorticoids and invasive fungal infections. Lancet 362: 1828-1838, 2003.

5. Rizwan A, Sarfaraz A, Jabbar A, Akhter J and Islam N: Case report: Nocardia infection associated with ectopic Cushings. BMC Endocr Disord 14: 51, 2014.

6. Sutton BJ, Parks GE, Manavi CK, Palavecino EL and Geisinger KR: Cushing's syndrome and nocardiosis associated with a pulmonary carcinoid tumor: Report of a case and review of the literature. Diagn Cytopathol 39: 359-362, 2011.

7. Momah $\mathrm{N}$ and Koroscil T: Occult ectopic adrenocorticotropic hormone secretion: Diagnostic dilemma and infective consequence. Clin Pract 2: e82, 2012.

8. Chrysanthidis T, Yavropoulou MP, Metallidis S, Mpakaimi I, Zempekakis P, Yovos JG and Nikolaidis P: Disseminated nocardiosis in ectopic adrenocorticotropic hormone syndrome. Endocrinologist 20: 286-287, 2010.

9. Chowdry RP, Bhimani C, Delgado MA, Lee DJ, Dayamani P, Sica GL and Owonikoko TK: Unusual suspects: Pulmonary opportunistic infections masquerading as tumor metastasis in a patient with adrenocorticotropic hormone-producing pancreatic neuroendocrine cancer. Ther Adv Med Oncol 4: 295-300, 2012.

10. Beinart GA, Hollander H and Rao R: Ectopic ACTH syndrome resulting in nocardiosis and acute respiratory failure. Hosp Physician 39: 49-54, 2003.

11. Higgins TL, Calabrese LH and Sheeler LR: Opportunistic infections in patients with ectopic ACTH-secreting tumors. Cleve Clin Q 49: 43-49, 1982.

12. Garcia LS (ed): Clinical Microbiology Procedures Handbook. Vol 2. 3rd edition. ASM Press, Washington, DC, 2010.

13. Natale RB, Yagoda A,Brown A,SingerC,StoverDandBajorunas D: Combined Pneumocystis carinii and Nocardia asteroides pneumonitis in a patient with an ACTH-producing carcinoid. Cancer 47: 2933-2935, 1981.

14. Petersen DP and Wong LB: Nocardia infection of the hand-case report. J Hand Surg Am 6: 502-505, 1981.

15. Kageyama A, Yazawa K, Ishikawa J, Hotta K, Nishimura K and Mikami Y: Nocardial infections in Japan from 1992 to 2001 including the first report of infection by Nocardia transvalensis. Eur J Epidemiol 19: 383-389, 2004.

16. Yu X, Han F, Wu J, He Q, Peng W, Wang Y, Huang H, Li H, Wang $\mathrm{R}$ and Chen $\mathrm{J}$ : Nocardia infection in kidney transplant recipients: Case report and analysis of 66 published cases. Transpl Infect Dis 13: 385-391, 2011.

17. Ejaz S, Vassilopoulou-Sellin R, Busaidy NL, Hu MI, Waguespack SG, Jimenez C, Ying AK, Cabanillas M, Abbara M and Habra MA: Cushing syndrome secondary to ectopic ACTH secretion: The University of Texas MD Anderson Cancer Center experience. Cancer 117: 4381-4389, 2011.

18. Isidori AM, Kaltsas GA, Pozza C, Frajese V, Newell-Price J, Reznek RH, Jenkins PJ, Monson JP, Grossman AB and Besser GM: The ectopic adrenocorticotropin syndrome: Clinical features, diagnosis, management and long-term follow-up. J Clin Endocrinol Metab 91: 371-377, 2006.

19. Ilias I, Torpy DJ, Pacak K, Mullen N, Wesley RA and Nieman LK: Cushing's syndrome due to ectopic corticotropin secretion: Twenty years' experience at the National Institutes of Health. J Clin Endocrinol Metab 90: 4955-4962, 2005.

20. Doi M, Sugiyama T, Izumiyama H, Yoshimoto T and Hirata Y: Clinical features and management of ectopic ACTH syndrome at a single institute in Japan. Endocr J 57: 1061-1069, 2010.

21. Bakker RC, Gallas PR, Romijn JA and Wiersinga WM: Cushing's syndrome complicated by multiple opportunistic infections. J Endocrinol Invest 21: 329-333, 1998.

22. Blackmon KN, Ravenel JG, Gomez JM, Ciolino J and Wray DW: Pulmonary nocardiosis: Computed tomography features at diagnosis. J Thorac Imaging 26: 224-229, 2011.

23. Chen J, Zhou H, Xu P, Zhang P, Ma S and Zhou J: Clinical and radiographic characteristics of pulmonary nocardiosis: Clues to earlier diagnosis. PloS One 9: e90724, 2014. 
24. Pecori Giraldi F, Moro M and Cavagnini F; Study Group on the Hypothalamo-Pituitary-Adrenal Axis of the Italian Society of Endocrinology: Gender-related differences in the presentation and course of Cushing's disease. J Clin Endocr Metab 88: 1554-1558, 2003.

25. Zhao K, Dong MJ, Sheng ZK, Liu KF, Yang SY, Liu ZF and Sheng JF: Elevated uptake of ${ }^{18} \mathrm{~F}-\mathrm{FDG}$ in PET/CT imaging of a nocardial pleural nodule. Clin Imaging 36: 383-385, 2012

26. Wilson JW: Nocardiosis: Updates and clinical overview. Mayo Clin Proc 87: 403-407, 2012.

27. Gombert ME, Berkowitz LB, Aulicino TM and duBouchet L: Therapy of pulmonary nocardiosis in immunocompromised mice. Antimicrob Agents Chemother 34: 1766-1768, 1990.
28. Ambrosioni J, Lew D and Garbino J: Nocardiosis: Updated clinical review and experience at a tertiary center. Infection 38: 89-97, 2010.

29. Moylett EH, Pacheco SE, Brown-Elliott BA, Perry TR, Buescher ES, Birmingham MC, Schentag JJ, Gimbel JF, Apodaca A, Schwartz MA, et al: Clinical experience with linezolid for the treatment of Nocardia infection. Clin Infect Dis 36: 313-318, 2003

30. Bhansali A, Dutta P, Bhat MH, Sinha SK and Nada R: Unusual opportunistic infection associated with endogenous Cushing syndrome. Endocrinologist 16: 125-127, 2006.

31. Newell-Price J, Bertagna X, Grossman AB and Nieman LK: Cushing's syndrome. Lancet 367: 1605-1617, 2006. 